\title{
Interplay of plasmon resonances in binary nanostructures
}

\author{
Y. Gu · Y. Wang · J. Li • O.J.F. Martin · Q. Gong
}

Received: 11 February 2009 / Revised version: 14 September 2009 / Published online: 21 November 2009

(C) Springer-Verlag 2009

\begin{abstract}
By introducing the difference permittivity ratio $\eta=\left(\epsilon_{2}-\epsilon_{0}\right) /\left(\epsilon_{1}-\epsilon_{0}\right)$, the Green matrix method for computing surface plasmon resonances is extended to binary nanostructures. Based on the near field coupling, the interplay of plasmon resonances in two closely packed nanostrips is investigated. At a fixed wavelength, with varying $\eta$ the resonances exhibit different regions: the dielectric effect region, resonance chaos region, collective resonance region, resonance flat region, and new branches region. Simultaneously, avoiding crossing and mode transfer phenomena between the resonance branches are observed. These findings will be helpful to design hybrid plasmonic subwavelength structures.
\end{abstract}

PACS 73.20.Mf $\cdot 78.67 .-\mathrm{n} \cdot 78.68 .+\mathrm{m}$

\section{Introduction}

Surface plasmon resonances (SPRs) of metallic nanoparticles have attracted great interest due to their applications in surface-enhanced Raman scattering [1], biosensor and nanometer plasmonic waveguide [2], optical antenna [3], solar cells [4], nonlinear optical frequency mixing [5-7] and

Y. Gu $(\bowtie) \cdot$ Y. Wang $\cdot$ J. Li · Q. Gong

State Key Laboratory for Mesoscopic Physics, Department

of Physics, Peking University, Beijing 100871, China

e-mail: ygu@pku.edu.cn

Q. Gong

e-mail: qhgong@pku.edu.cn

O.J.F. Martin

Nanophotonics and Metrology Laboratory, Swiss Federal Institute of Technology Lausanne (EPFL), EPFL-STI-NAM, ELG Station

11, 1015 Lausanne, Switzerland so on. With the development of nanofabrication and nanolithography techniques, various metallic nanoparticles, such as nanospheres, nanoshells, nanorices, nanorings, nanostars, nanocages, and nanotriangles, have been successfully fabricated, exhibiting a variety of resonant behaviors [2]. The resonance frequencies of nanoshells can be tuned from visible to infrared frequencies by adjusting the core-shell ratio [2], and the nanocrescents have shown multiple, adjustable resonances and stronger enhanced near fields due to their unique structure with tips and ring [8]. Simultaneously, the red and blue shifts of resonances, based on the near field coupling among nanoparticles, have been reported [9-12], as well as the scaling rules of these shifts $[13,14]$. However, the interplay of SPR among nanoparticles with different material parameters is scarcely studied. The goal of this work is to explore the resonance combination of surface plasmon in binary nanostructures.

The Green tensor method has successfully solved the optical near field of the isolated nanostructures [15]. And the Green function formalism can give the dielectric resonance information only in the quasistatic limit [16]. Recently, by combining the Green tensor method with the Green function formalism, we have developed the Green matrix method (GMM) to deal with the surface plasmon resonances and near field of the arbitrary shaped subwavelength metallic structures [17]. Instead of the traditional methods which give the resonance peaks in the wavelength region, by using the resonance capacity of surface plasmon [17], GMM can give the resonance spectrum with respect to a set of dielectric permittivities. Here the GMM can be used to design novel plasmonic, or hybrid plasmonic-dielectric, structures with desired resonance properties. This is different from all conventional numerical techniques, which can only compute the response of a given structure and not determine the mater- 
ial parameters, required for this structure to exhibit specific resonances.

In the following, by introducing the difference permittivity ratio $\eta=\left(\epsilon_{2}-\epsilon_{0}\right) /\left(\epsilon_{1}-\epsilon_{0}\right)$, we extended the Green matrix method from single-component to two-component structures with permittivities $\epsilon_{1}$ and $\epsilon_{2}$ in a homogeneous background $\epsilon_{0}$. Based on the formalism and resonance capacity concept [17], we investigate the interplay of surface plasmon resonances in two closely located nanostrips with the same geometry. With varying $\eta$ at a fixed wavelength, the resonance branches experience different regions: the $d i$ electric effect region, resonance chaos region, collective resonance region, resonance flat region, and new branches region. When $\eta<0$, i.e., one nanostrip is dielectric and the other is metallic, the resonances are dominated by the metallic nanostrip, and there are small shifts and splits of resonances due to the influence of the dielectric. For $\eta>0$, i.e., both nanostrips are metallic, several resonance branches with large shifts and splits appear and generally one or two strong resonances dominate, exhibiting a collective resonance or a one nanostrip-dominated resonance. Simultaneously, both for $\eta<0$ and $\eta>0$, avoiding crossing and mode transfer phenomena between the resonance branches are observed. Other combined nanostructures, such as two nanostructures with different geometry are also studied. When $\eta<0$, almost the same behavior is found as previously. However, for $\eta>0$, many SPR branches appear, and it looks like the strong resonances are destroyed into many weak ones.

\section{Green matrix method for two-component subwavelength structures}

Consider the arbitrary shaped two-component subwavelength structures (or nanostructures in the optical frequency region) with the dielectric permittivities $\epsilon_{1}(\mathbf{r}, \omega)$ and $\epsilon_{2}(\mathbf{r}, \omega)$, embedded in the homogeneous bulk material $\epsilon_{0}(\omega)$, as shown in Fig. 1. These nanostructures are not necessarily connected, but outside the structures, the tensors $\epsilon_{s i}(\mathbf{r}, \omega)\left(=\epsilon_{i}(\mathbf{r}, \omega)-\epsilon_{0}(\omega)\right.$ for $i=1$ or 2$)$ are vanishing. Dielectric permittivities $\epsilon_{1}(\mathbf{r}, \omega)$ and $\epsilon_{2}(\mathbf{r}, \omega)$ are generally complex and frequency dependent. If a monochromatic field
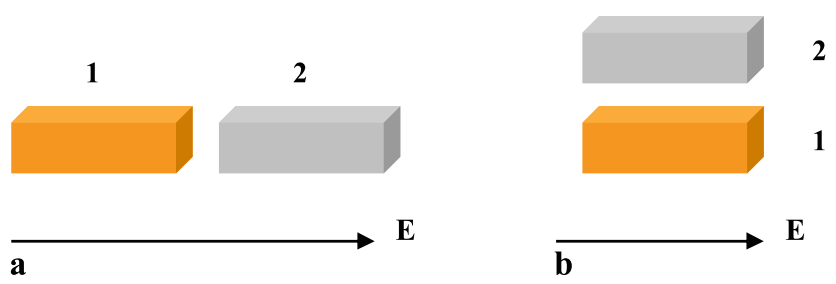

Fig. 1 Scheme of two $60 \times 20 \times 20 \mathrm{~nm}^{3}$ nanostrips for (a) the parallel case and (b) the perpendicular case
$E^{0}(\mathbf{r}) e^{-i \omega t}$ impinges on the system, the scattered field $E(\mathbf{r})$ is a solution of the wave equation

$$
\begin{gathered}
-\nabla \times \nabla \times E(\mathbf{r})+k^{2} \epsilon_{0}(\omega) E(\mathbf{r}) \\
+k^{2} \epsilon_{s i}(\mathbf{r}, \omega) E(\mathbf{r})=0
\end{gathered}
$$

where $k$ is the vacuum wave number.

By introducing the Green operator $G^{0}\left(\mathbf{r}, \mathbf{r}^{\prime}, \omega\right)$ associated with the bulk material,

$\nabla \times \nabla \times G^{0}\left(\mathbf{r}, \mathbf{r}^{\prime}, \omega\right)-k^{2} \epsilon_{0}(\omega) G^{0}\left(\mathbf{r}, \mathbf{r}^{\prime}, \omega\right)=I$

and $G\left(\mathbf{r}, \mathbf{r}^{\prime}, \omega\right)$ associated with the scattering nanocluster [18],

$$
\begin{aligned}
\nabla & \times \nabla \times G\left(\mathbf{r}, \mathbf{r}^{\prime}, \omega\right)-\left[k^{2} \epsilon_{0}(\omega)+k^{2} \epsilon_{s i}(r, \omega)\right] \\
& \times G\left(\mathbf{r}, \mathbf{r}^{\prime}, \omega\right)=I,
\end{aligned}
$$

where $I$ is the unit operator and following some transformations, the Lippmann-Schwinger equation [15, 19] of the field $E(\mathbf{r})$ at any point $\mathbf{r}$ can be extended to the twocomponent subwavelength clusters:

$$
\begin{aligned}
E(\mathbf{r})= & E^{0}(\mathbf{r})+k^{2} \int_{c_{1}} d \mathbf{r}^{\prime} G^{0}\left(\mathbf{r}, \mathbf{r}^{\prime}, \omega\right) \epsilon_{s 1}(\omega) \cdot E\left(\mathbf{r}^{\prime}\right) \\
& +\eta k^{2} \int_{c_{2}} d \mathbf{r}^{\prime} G^{0}\left(\mathbf{r}, \mathbf{r}^{\prime}, \omega\right) \epsilon_{s 1}(\omega) \cdot E\left(\mathbf{r}^{\prime}\right),
\end{aligned}
$$

where $C=C_{1} \cup C_{2}$ denotes the cluster subspace, and $\eta=$ $\left(\epsilon_{2}-\epsilon_{0}\right) /\left(\epsilon_{1}-\epsilon_{0}\right)$ is called the difference permittivity ratio [20]. In the subspace $C_{1}$ or $C_{2}, \epsilon_{1}(\mathbf{r}, \omega)$ or $\epsilon_{2}(\mathbf{r}, \omega)$ is simplified as $\epsilon_{1}(\omega)$ or $\epsilon_{2}(\omega)$. Green's tensor in a three dimensional system is analytically known [18]:

$$
\begin{aligned}
G^{0}\left(\mathbf{r}, \mathbf{r}^{\prime}, \omega\right)= & \left(\mathbf{I}-\frac{1-i k_{0} R}{k_{0}^{2} R^{2}} \mathbf{I}-\frac{-3+3 i k_{0} R+k_{0}^{2} R^{2}}{k_{0}^{2} R^{4}} \mathbf{R R}\right) \\
& \times \frac{\exp \left[i k_{0} R\right]}{4 \pi R},
\end{aligned}
$$

where $R=|\mathbf{R}|=\left|\mathbf{r}-\mathbf{r}^{\prime}\right|$ and $k_{0}^{2}=k^{2} \epsilon_{0}(\omega)$. Equation (4) means that the field inside the nanocluster can be solved selfconsistently in the cluster subspace, while the field outside the cluster can be expressed explicitly through the Green propagator $G^{0}\left(\mathbf{r}, \mathbf{r}^{\prime}, \omega\right)$. Let us note the similar field expression in (7) of [16], where the Green function formalism was established to obtain the dielectric resonance spectrum and local field distribution in the quasistatic limit. With almost the same procedure as in [16], the Green matrix method has been established to deal with surface plasmon resonances of arbitrary shaped nanocluster beyond the quasistatic limit, [17]. Here, starting from (4), we extend the GMM from single-component to two-component subwavelength structures embedded in the bulk material. 
If the nanocluster subspace is discretized into $N$ pieces with volume $\delta V$ (in principle $\delta V \ll V$ ), let $\widetilde{G^{0}}\left(\mathbf{r}, \mathbf{r}^{\prime}, \omega\right)=$ $\delta V k^{2} G^{0}\left(\mathbf{r}, \mathbf{r}^{\prime}, \omega\right) \delta_{\mathbf{r}^{\prime}, \mathbf{r}_{1}}+\eta \delta V k^{2} G^{0}\left(\mathbf{r}, \mathbf{r}^{\prime}, \omega\right) \delta_{\mathbf{r}^{\prime}, \mathbf{r}_{2}}$ for $\mathbf{r}_{1} \in$ $C_{1}$ and $\mathbf{r}_{2} \in C_{2}$ in the clusters subspace $C=C_{1} \cup C_{2}$, (4) becomes

$$
\sum_{\mathbf{r}^{\prime} \in C}\left[\epsilon_{s 1}(\omega) \widetilde{G^{0}}\left(\mathbf{r}, \mathbf{r}^{\prime}, \omega\right)-\delta_{\mathbf{r}, \mathbf{r}^{\prime}}\right] \widetilde{E}(\mathbf{r})=-\widetilde{E^{0}}(\mathbf{r})
$$

Here the Green tensor $\widetilde{G^{0}}\left(\mathbf{r}, \mathbf{r}^{\prime}, \omega\right)$ is extending all over the points in $C$. The Green matrix $\widetilde{G}^{0}$ is a $3 N \times 3 N$ square matrix. Equation (6) can be rewritten the following form:

$\sum_{\mathbf{r}^{\prime} \in C}\left[\widetilde{G^{0}}\left(\mathbf{r}, \mathbf{r}^{\prime}, \omega\right)-s \mathbf{I}\right] \widetilde{E}(r)=\frac{-\widetilde{E^{0}}(\mathbf{r})}{\epsilon_{s 1}(\omega)}$

where $s=\frac{1}{\epsilon_{s 1}(\omega)}=\frac{1}{\epsilon_{1}(\omega)-\epsilon_{0}(\omega)}$. Since $\widetilde{G^{0}}$ is symmetrical, mathematically, there are $3 N$ real eigenvalues. Physically, only those eigenvalues with the large residue of electric field correspond to the strong resonances, which are selected by the calculations of the resonance capacity [17]. Let us emphasize a major difference between the approach proposed here and conventional calculations. Usually, (6) is solved to compute the scatted field $\widetilde{E}(\mathbf{r})$ for a given illumination $\widetilde{E}^{0}(\mathbf{r})$ and a given scattering system. By considering (7) and solving an eigenvalue problem, we are able to determine the conditions where the system will exhibit a resonant behavior. The mathematical eigenvalues depend on the numerical discretization, while the physical resonances do not. Furthermore, the regions of eigenvalue $s_{n}$ are clearly related to the ratio $\eta$. Different from the single-component nanostructure case, where most of the eigen dielectric permittivities $\epsilon_{n}(r, \omega)\left(=\frac{1}{s_{n}}+\epsilon_{0}(\omega)\right)$ fall into the negative real axis [17], here at least one of the eigen dielectric permittivities, $\epsilon_{1 n}(\omega)\left(=\frac{1}{s_{n}}+\epsilon_{0}(\omega)\right)$ or $\epsilon_{2 n}(\omega)\left(=\eta \cdot \frac{1}{s_{n}}+\epsilon_{0}(\omega)\right)$, should have a negative value. Hence it is natural that the cooperation of positive permittivity (here $\epsilon_{0}=1.0$ ) and negative permittivity $\epsilon_{1 n}(\omega)$ or $\epsilon_{2 n}(\omega)$ leads to the resonances of surface plasmon. Different from the quasistatic limit where the dielectric resonances only relate to the dielectric permittivities of cluster and reference media [16], here with the retardation effect, the SPRs are directly related to the wave number $k$.

Generally we assume that the field $\widetilde{E}(\mathbf{r})($ a $3 N \times 1$ matrix) in the cluster subspace has the form:

$\widetilde{E}(\mathbf{r})=\sum_{n=1}^{3 N} A_{n}(s) \cdot R_{n}$

where $R_{n}$ is the right eigenvector of the $n$th eigenvalue of $\widetilde{G^{0}}$. By substituting (8) in (7) and multiplying the left eigenvector $L_{n}\left(=R_{n}^{T}\right)$ in $(7), A_{n}(s)$ is obtained and $\widetilde{E}(\mathbf{r})$ in the nanocluster reads

$\widetilde{E}(\mathbf{r})=\sum_{n=1}^{3 N} \frac{L_{n} \cdot \widetilde{E^{0}}(\mathbf{r})}{\epsilon_{s 1}(\omega)\left(s-s_{n}\right)} \cdot R_{n}$.

For any point $\mathbf{r}$ outside the nanocluster, we have

$$
\begin{aligned}
E(\mathbf{r})= & E^{0}(\mathbf{r})+k^{2} \sum_{n=1}^{3 N} \frac{L_{n} \cdot \widetilde{E^{0}}(\mathbf{r})}{\left(s-s_{n}\right)} \\
& \cdot\left[\left(\sum_{\mathbf{r}^{\prime} \in C_{1}} G^{0}\left(\mathbf{r}, \mathbf{r}^{\prime}, \omega\right)+\eta \sum_{\mathbf{r}^{\prime} \in C_{2}} G^{0}\left(\mathbf{r}, \mathbf{r}^{\prime}, \omega\right)\right) \cdot R_{n}\right] .
\end{aligned}
$$

Until now, for any $\epsilon_{1}(\omega)$ and $\epsilon_{2}(\omega)$, the optical near field can be expressed by the Green tensor $G^{0}\left(\mathbf{r}, \mathbf{r}^{\prime}, \omega\right)$, eigenvalues $s_{n}$ and eigenvectors $R_{n}, L_{n}$ of the Green matrix $\widetilde{G^{0}}$. Obviously, the far field can also be directly given from the above, (9) and (10).

When $s$ approaches one of the eigenvalues $s_{n}$, i.e., $s \rightarrow$ $s_{n}$, the electric field diverges to infinity in the case of no loss. Only those eigenstates (or resonances) with strong electric near field are physically meaningful and have some applications [1-7]. To select those SPRs, we have defined the resonance capacity based on the internal energy of the nanostructures [17]. In two-component nanostructures, for each $s_{n}$, the resonance capacity of component $i$ is

$C_{i n}=\frac{\int_{C_{i}} d \mathbf{r}^{\prime}\left|\epsilon_{i n}\right| \cdot\left[\operatorname{res}\left|E\left(\mathbf{r}^{\prime}\right)\right|\right]_{n}^{2}}{\int_{C_{i}} d \mathbf{r}^{\prime}\left|\epsilon_{0}(\omega)\right| .\left|E^{0}\left(\mathbf{r}^{\prime}\right)\right|^{2}}$

where the residue of the field is $\left[\operatorname{res}\left|E\left(\mathbf{r}^{\prime}\right)\right|\right]_{n}=$ $\left|\frac{L_{n} \cdot E^{0}\left(\mathbf{r}^{\prime}\right)}{\epsilon_{1 n}(\mathbf{r}, \omega)} \cdot R_{n}\right|, \epsilon_{1 n}=\frac{1}{s_{n}}+\epsilon_{0}(\omega), \epsilon_{2 n}=\frac{\eta}{s_{n}}+\epsilon_{0}$, and $i=1,2$. Large resonance capacity values usually represent the strong resonances with a more enhanced near field. Conceptually, the extinction peaks of far field should correspond to the SPRs with high value of resonance capacity. In the following, by performing some numerical calculations, we investigate the evolution of the resonance capacity and near field distributions for varying difference permittivity ratios $\eta$.

\section{Surface plasmon resonances and near field distributions}

The SPR properties of two closely packed metallic nanoparticles with the same geometry and same dielectric permittivity have been widely investigated [9-14]. When the central line of both particles is parallel to the polarization of the electric field, a red shift of the resonance wavelength is found; while, when the central line of both particles is perpendicular to the polarization of the electric field, a blue 
shift is found [9-12]. It is known that the resonance occurs at the matching conditions for the geometry, incident light, metallic permittivity and dielectric environment. Hence the position and intensity of the resonances can be affected by its neighboring nanoparticles embedded in the dielectric environment. In the following, SPR and near field distributions of two nanostrips are studied as a function of the difference permittivity ratio $\eta$.

As shown in Fig. 1(a), when the central line of both nanostrips is parallel to the polarization of external electric field, we call it the parallel case; while, in Fig. 1(b), when their central line is perpendicular to the polarization of electric field, we call it the perpendicular case. Here the input wavelength is $\lambda=632.8 \mathrm{~nm}$, the size of each nanostrip is $60 \times 20 \times 20 \mathrm{~nm}^{3}$, and both gaps are $10 \mathrm{~nm}$ with the discretization mesh $5 \mathrm{~nm}$. The electric field is propagating along the $z$ direction and polarized along the $x$ direction. For the calculations of the near field distributions associated with resonances, we have selected the $x y$ plane $10 \mathrm{~nm}$ above the nanostrips and included an imaginary parts of $0.15 i$ in the parallel case and $0.05 i$ in the perpendicular case. When the incident wavelength is $\lambda=632.8 \mathrm{~nm}$, for a single $60 \times 20 \times 20 \mathrm{~nm}^{3}$ nanostrip, there is only one resonance at $\epsilon_{1}(\omega) \approx-11.7$, corresponding to the gold material at this wavelength [17].

Before giving a detailed discussion, let us outline the main results of this section, as well as clarify the mode classification and the nomination of resonance branches in the $\eta-s$ diagrams in Figs. 2 and 4, where $s$ is one of the eigenvalues of the Green matrix $\widetilde{G^{0}}$ of the system. Due to the geometrical symmetry of both nanostrips, we only need to consider here the resonances dominated by nanostrip 1 . The 4 main branches represent 4 types of resonances associated to nanostrip 1 , which intensities are expressed as $C_{1}$. When $\eta<0$, i.e. nanostrip 2 is a dielectric being closely located to the resonant nanostrip 1, resonances are barely affected by the dielectric nanoparticle, hence this is the dielectric effect region, flagged as branch 1 . However, when nanostrip 2 is metallic, i.e., $\eta>0$, there will be a large influence on the resonant properties of metallic nanostrip 1 due to the existence of free electrons in metallic nanostrip 2 . When $\eta \in[0,0.95]$, the resonance capacity of nanostrip 1 is very sensitive to $\eta$, this is called resonance chaos region, marked as subbranch 21. For $\eta \in[0.65,1.3]$, i.e., when two metallic nanostrips have comparable dielectric permittivities, collective resonances occur and this is called the collective resonance region, marked as subbranch 22 . When $\eta>1.5$, the resonance capacity becomes large and stable, which is called the resonance flat region, marked as subbranch 23. Finally, when $\eta>3.0$, the system enters the new branches region, where the new resonance branches 3 and 4 appear. Except the disordered subbranch 21 , for one subbranch, there is always a smooth curve of resonance capacity which starts from zero, passes a maximum value, and finally reaches to zero again; and generally one branch is up with another branch down, as shown in Fig. 2(b). For a large difference between both permittivities, generally we observe that the one particle dominates the resonance; however, it is not always the metallic particle with the largest permittivity determines the resonances, sometimes the smallest one dominates. In the above mentioned regions characterized by different values of $\eta$, a variety of resonant behaviors are visible, including the avoided crossing between resonant branches, collective resonances of two particles, and the mode transfer phenomenon. In the following, we discuss these different resonant behaviors for parallel and perpendicular geometries.

\subsection{Parallel case}

Dielectric effect region Figure 2 displays 4 main resonance branches of nanostrip 1 , the resonance capacity $C_{1}$ and $C_{2}$ of nanostrip 1 and nanostrip 2, and the corresponding $\epsilon_{1}$ and $\epsilon_{2}$ as a function of $\eta$. It is flagged by resonance branch 1 , where $\eta \in[-2.3,0)$, and $\epsilon_{1} \in[-11.36,-10.12]$, $\epsilon_{2} \in[26.58,1]$. In this region, resonances are dominated by nanostrip 1 and only marginally affected by the dielectric nanostrip 2 since the electromagnetic wave can penetrate the dielectric. When a resonance occurs, the dielectric permittivity $\epsilon_{1}$ experiences a little shift due to the existence of $\epsilon_{2}$, and there is a little increment in the resonance capacity $C_{1}$ with increasing $\epsilon_{2}$. Of course, the resonance capacity $C_{2}$ is very small since the nanostrip 2 is dielectric. At about $\eta=-1.56$ in branch 1 , there is avoiding crossing between subbranches 11 and 12 in Fig. 2(a). In Fig. 2(b), we can see a minimum of resonance capacity at $\eta=-1.56$. Simultaneously, near $\eta=-1.56$, when the branch 11 is transferred to branch 12, the splitting of SPR is also found, i.e., for one $\eta$, there are two close large resonances. The near field distributions around the area $A$ are illustrated in Fig. 3(a). It is seen that, with decreasing $\eta$ from -1.5 to -1.6 , there is a depression in the intensity of near field at $\eta=-1.56$. In all three parameters, the near fields are localized within the nanostrip 1. It is noted that this depression only happens when $\left|\epsilon_{2}\right|>\left|\epsilon_{1}\right|$, which means that the dielectric nanostrip 2 with a large positive dielectric constant can also have an important effect on the nanostrip 1. However, in the optical frequencies, it is difficult to find the corresponding experimentally accessible materials.

Resonance chaos region It is flagged by subbranch 21 , where $\eta \in[0,0.95]$, and $\epsilon_{1} \in[-11.23,-9.60], \epsilon_{2} \in[-0.47$, $-9.0726]$. In this region, the resonance capacity of nanostrip 1 is very sensitive to $\eta$, although we could not find the explicit features, the resonances are clearly dominated by nanostrip 1 with the conditions $\left|\epsilon_{1}\right|>\left|\epsilon_{2}\right|$. We think that 

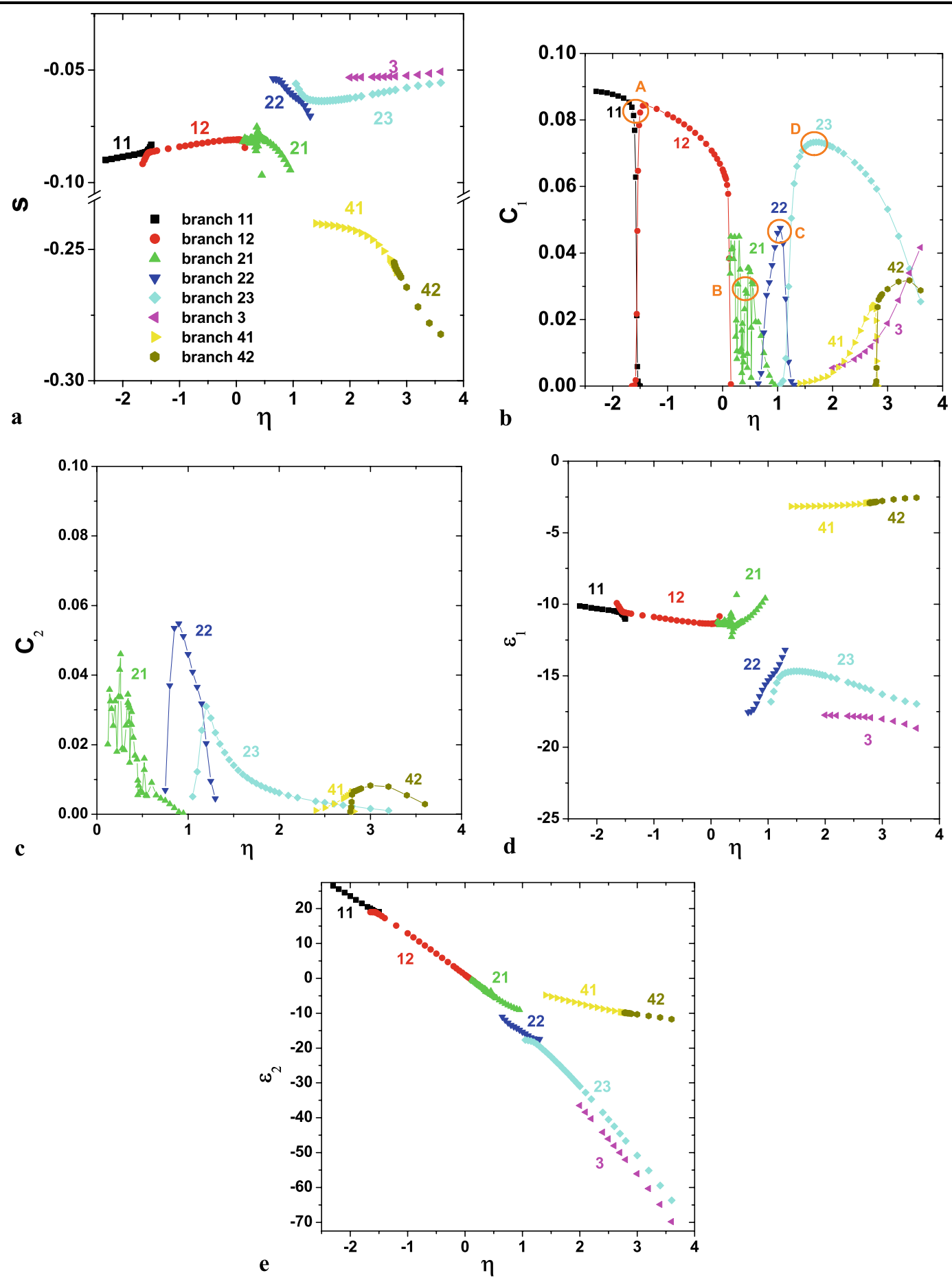

Fig. 2 Resonance combination for the parallel case with varying $\eta$. (a) Resonance branches $s$, (b) resonance capacity for nanostrip 1, (c) resonance capacity for nanostrip 2, (d) eigen dielectric permittiv-

this "chaotic" phenomenon comes from very high density of branches. It is not easy to classify each resonance subbranch and we have only selected those with the strongest resonances. Our numerical calculations for symmetrical three nanostrips also support that for a small $\eta$ region with $\eta>0$ ity $\epsilon_{1}$, and (e) eigen dielectric permittivity $\epsilon_{2}$. Here 4 main branches represent 4 types of resonances referred to nanostrip 1, which intensities are expressed as the $C_{1}$ for nanostrip 1 and $C_{2}$ for nanostrip 2

this phenomenon exists [21]. Figure 3(b) illustrates the near field distributions at $\eta=-0.30,0.36$, and 0.40 , and near field sensitivity to $\eta$ is clearly shown. Compared with the branch 1 , the total near fields are weaker, but the near field in nanostrip 2 is stronger. Sometimes the resonance capacity 


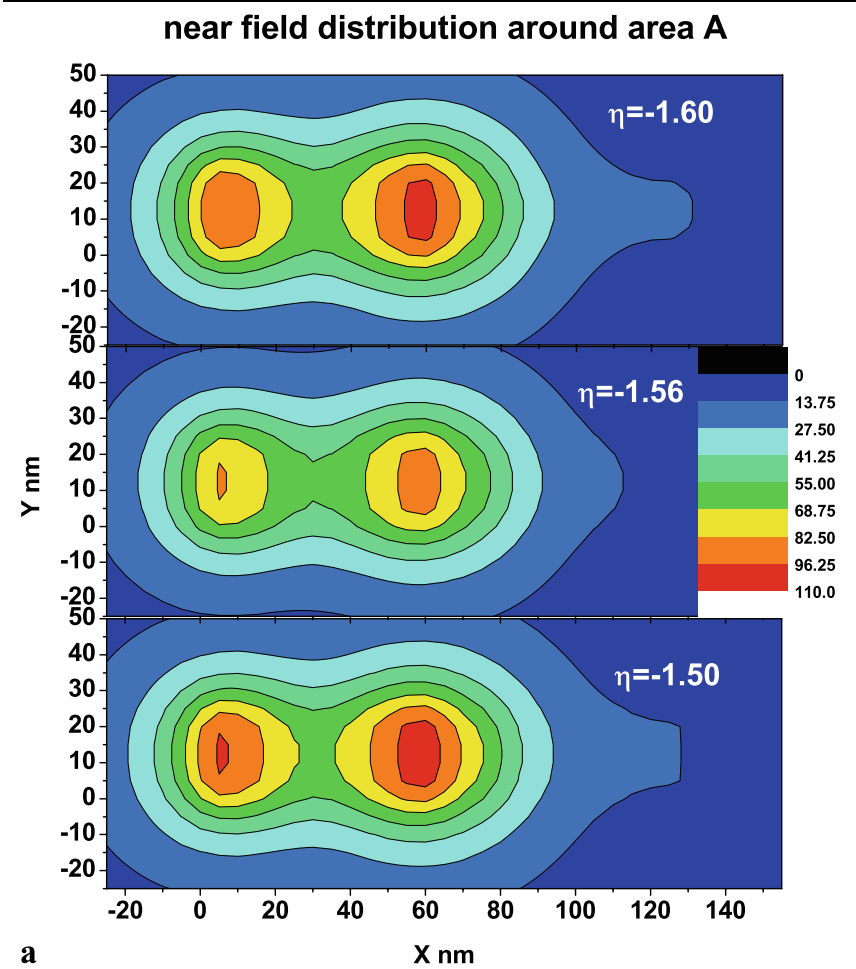
near field distribution around area $\mathrm{C}$
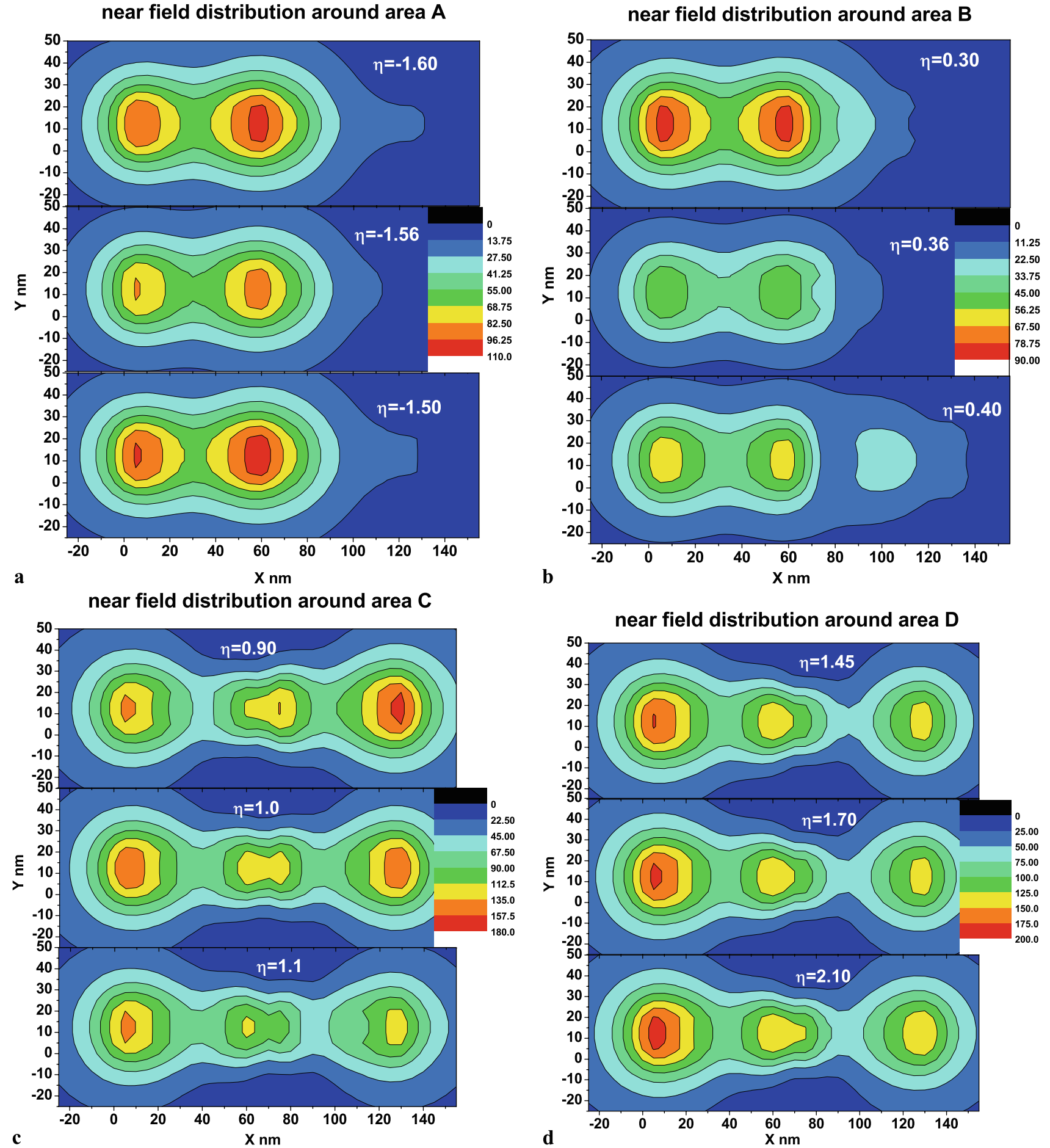

Fig. 3 Near field distributions above $10 \mathrm{~nm}$ of nanostrips around (a) area $A,(\mathbf{b})$ area $B,(\mathbf{c})$ area $C$, and (d) area $D$. Here the scale means the intensity of near field gathering at resonances caused by the same imaginary part $0.15 i$

$C_{2}$ can be comparable with $C_{1}$ of nanostrip 1 , as shown in Fig. 2(c).

Collective resonance region When the dielectric permittivities of two nanostrips have comparable values, the fol- lowing characterization of the collective resonances can be made. That is subbranch 22 , where $\eta \in[0.65,1.3]$, and $\epsilon_{1} \in[-17.55,-13.19], \epsilon_{2} \in[-11.06,-17.44]$. As shown in Figs. 2(d) and 2(e), when $\eta=1, \epsilon_{1}=\epsilon_{2}=-15.32$, if the permittivity region is mapped to the wavelength region, 
compared with the result of a single nanostrip, there is a large red shift in the resonance frequency [9-12]. For $\eta=$ 0.9 , when resonances happen, $\left|\epsilon_{1}\right|<\left|\epsilon_{2}\right|$ and the resonance capacity of nanostrip 2 is larger than that of nanostrip 1; while for $\eta=1.1$, when resonances happen, $\left|\epsilon_{1}\right|>\left|\epsilon_{2}\right|$ and the resonance capacity of nanostrip 1 is larger than that of nanostrip 2. The detailed near field distributions are illustrated in Fig. 3(c), corresponding to the area $C$ in Fig. 2(b). For $\eta=0.9$, the electric field near nanostrip 2 is stronger than that of nanostrip 1. In contrast, for $\eta=1.1$, the electric field near nanostrip 1 is stronger. It is noted that for $\eta=1$ the strong near fields are distributed in the outside of two nanostrips, rather than in the center of them. Further numerical results indicate that if we choose the $x y$ plane within the metal, the electric field is enhanced within the gap, in line with nanoantenna results [3].

Resonance flat region After the collective resonance region, the resonance will enter the resonance flat region, marked as branch 23 . When $\eta>1.5$, the values of resonance capacity become high and stable. In this region, $\eta \in[1.05,3.6]$, and $\epsilon_{1} \in[-14.68,-16.97], \epsilon_{2} \in[-17.70$, $-63.67]$. We found that, though now $\left|\epsilon_{1}\right|<\left|\epsilon_{2}\right|$, the resonances of nanostrip 1 dominate the system. This is different from what was found in branch 21 , where $\left|\epsilon_{1}\right|>\left|\epsilon_{2}\right|$ and still the nanostrip 1 dominated. From the resonance capacity in Fig. 2(b), it is seen that in a very wide region from $\eta=1.35$ to $\eta=2.8$, there is a large near field distribution and not much changes in the average near field. To verify this point, Fig. 3(d) illustrates the near field distributions around the area $D$. It is also found that in branch 23 there are still considerable electric fields near nanostrip 2.

New resonance branches When $\eta>3.0$, near branch 23 , the new branches 3 and 4 emerge. Branch 23 disappears and main resonant behavior is determined by branches 3 and 4. Especially, at $\eta=3.4$, the resonance capacities of surface plasmon in branch 23 , branch 3 and branch 4 almost have the same values, exhibiting resonance splitting or multiple resonance phenomena. Previously, multiple resonance of surface plasmon has been reported in the nanocrescent and C-type structures [22, 23]. Moreover, when $\eta \in[2.0,3.5]$, there are three branches: for branch 23, $\epsilon_{1} \in[-14.99,-16.97], \epsilon_{2} \in[-30.98,-63.67]$; for branch $3, \epsilon_{1} \in[-17.76,-18.67], \epsilon_{2} \in[-36.51,-69.81]$; and for branch $4, \epsilon_{1} \in[-3.13,-2.54], \epsilon_{2} \in[-7.26,-11.76]$. It is found that near $\eta=2.8$ the avoiding of crossing and mode transfer phenomena between branch 41 and branch 42 appear. We noted the same phenomena of dielectric resonance occurring in the quasistatic limit [20, 24]. When $\eta$ is very large, the resonant dielectric permittivities of both nanostrips go beyond the optical frequency region and are of limited practical use in optics.

\subsection{Perpendicular case}

In the parallel case, there are 4 main branches, including 8 subbranches. Except for the Resonance chaos region flagged by branch 21 , there are clearly boundaries between other subbranches, as well as smooth resonance capacity curves. While, for the perpendicular case, the situation is very different. Roughly speaking, there are 4 main branches, but it is very difficult to distinguish those subbranches. Branch 1 is still the dielectric effect region, branch 2 roughly includes the resonance chaos region, collective resonance region, and resonance flat region, and branches 3 and 4 belong to the new branches regions. Compared with the parallel case, the resonant behavior of the perpendicular case is more complicated.

Figure 4 displays 4 resonance branches of nanostrip 1, the resonance capacities $C_{1}$ and $C_{2}$ of nanostrip 1 and nanostrip 2, and the corresponding $\epsilon_{1}$ and $\epsilon_{2}$ in the perpendicular case as a function of $\eta$. This paragraph is focused on the dielectric effect region and new branches region, as shown in Figs. 4(a) and 4(b). In the following, the resonance regions in branch 2 are emphasized individually. It is similar to what was found in the parallel case, in the dielectric effect region, the dielectric nanostrip 2 has only a small influence on the resonance position and intensity. In branch 1, we have found avoiding of crossing twice with decreasing $\eta$. In the new branches region, branches 3 and 4 have a similar behavior to that in the parallel case. Moreover, the area $E$ in branch 4 has taken on the interesting mode transfer behavior. As shown in Fig. 5(a), at $\eta=2.20$ (with $\epsilon_{1}=-3.48057, \epsilon_{2}=-8.85725$ ) the strong near field concentrates on above the nanostrip 1 , via $\eta=2.30$ (with $\epsilon_{1}=-3.34877, \epsilon_{2}=-9.00216$ and $\left.\epsilon_{1}=-3.45445, \epsilon_{2}=-9.24524\right)$ where both modes have the weaker near field distributions, and finally at $\eta=2.40$ (with $\epsilon_{1}=-3.32179, \epsilon_{2}=-9.37229$ ) the strong near fields are transferred from nanostrip 1 to nanostrip 2 . This is a typical mode transfer phenomenon, which is also reported in the quasistatic dielectric resonance spectra [24]. The strong near field above nanostrip 2 for $\eta=2.40$ can be explained as follows. Though on average the nanostrip 1 has gathered more electric fields, the strongest field parts are within the gap between nanostrip 1 and nanostrip 2, and the collective effect of nanostrip 1 and nanostrip 2 leads to the near field distribution shown in Fig. 5(a). Simultaneously, we also found that the resonance capacity values of nanostrip 1 in the perpendicular case are larger than those in the parallel case. A simple explanation may be that they have suffered different types of near field effects from nanostrip 2.

The resonant character of branch 2 is individually exhibited in Fig. 4(c). Though some similar properties exist in the parallel and perpendicular cases, they are also many differences. Different from that in the parallel case, the whole 

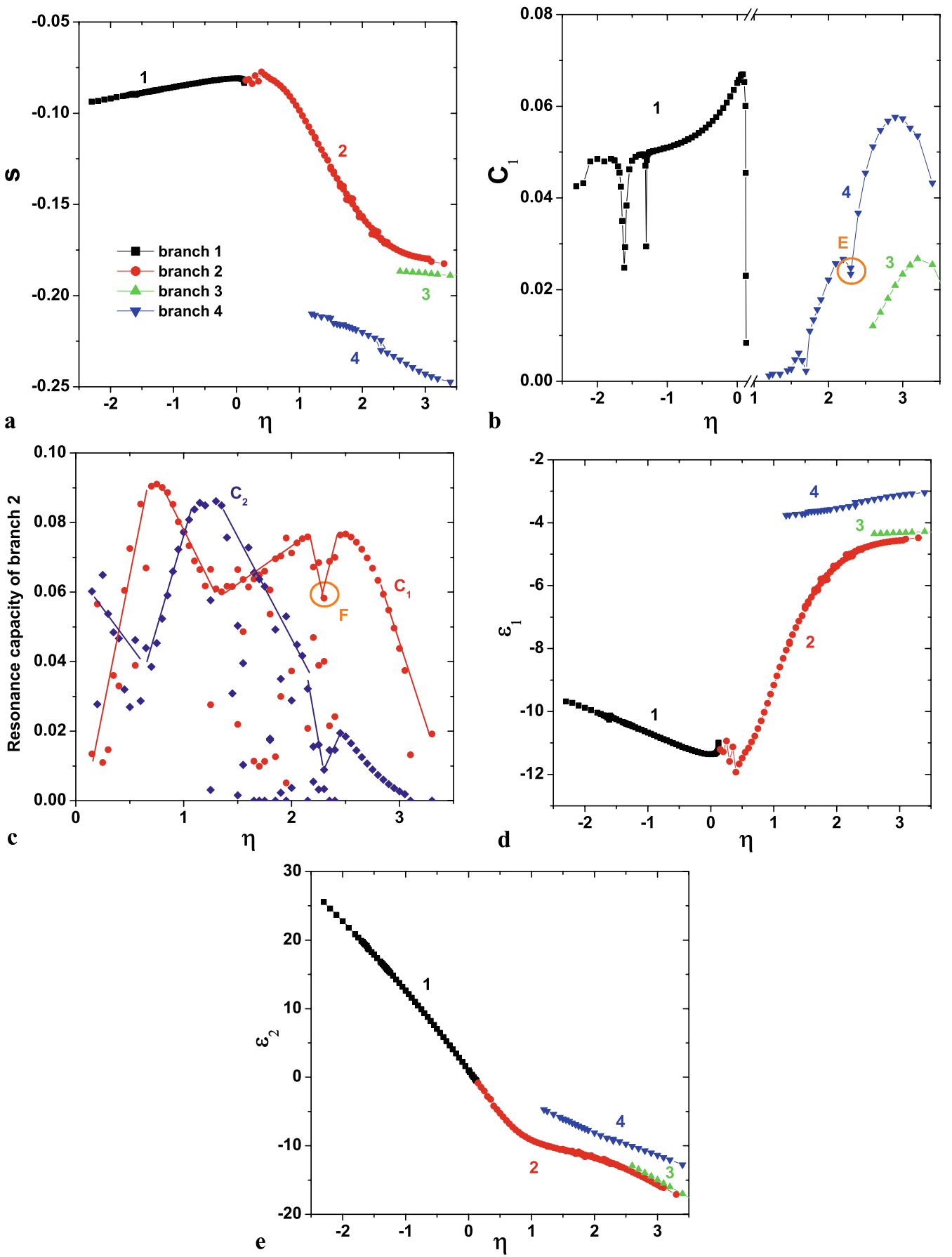

Fig. 4 Resonance combination for the perpendicular case with varying $\eta$. (a) Resonance branches, (b) resonance capacity for branches 1,3 , and 4 , (c) resonance capacity for branch 2 , (d) eigen dielectric permittivity $\epsilon_{1}$, and (e) eigen dielectric permittivity $\epsilon_{2}$. Here the main

branch 2 is almost connected. Roughly speaking, at the region $\eta \in[0.0,0.5]$ and at the region $\eta \in[0.6,1.5]$, similar to the parallel case, the resonance chaos region and collective resonance region appear. When $\eta=1.0$ with $\epsilon_{1}=$ $\epsilon_{2}=-9.16$, if the permittivity region is mapped to the wavelength region, a blue shift of resonance frequency should branches represent the different types of resonances referred to nanostrip 1, which intensities are expressed as the $C_{1}$ for nanostrip 1 and $C_{2}$ for nanostrip 2

be found [9-12]. One of the important differences is that with varying $\eta$, resonance splitting exists everywhere, which means that multi-branches coexist. While, in the parallel case, except for the resonance chaos region, two branches coexist in most cases. Because in the perpendicular case we could not clearly distinguish those subbranches, in Fig. 4(c) 

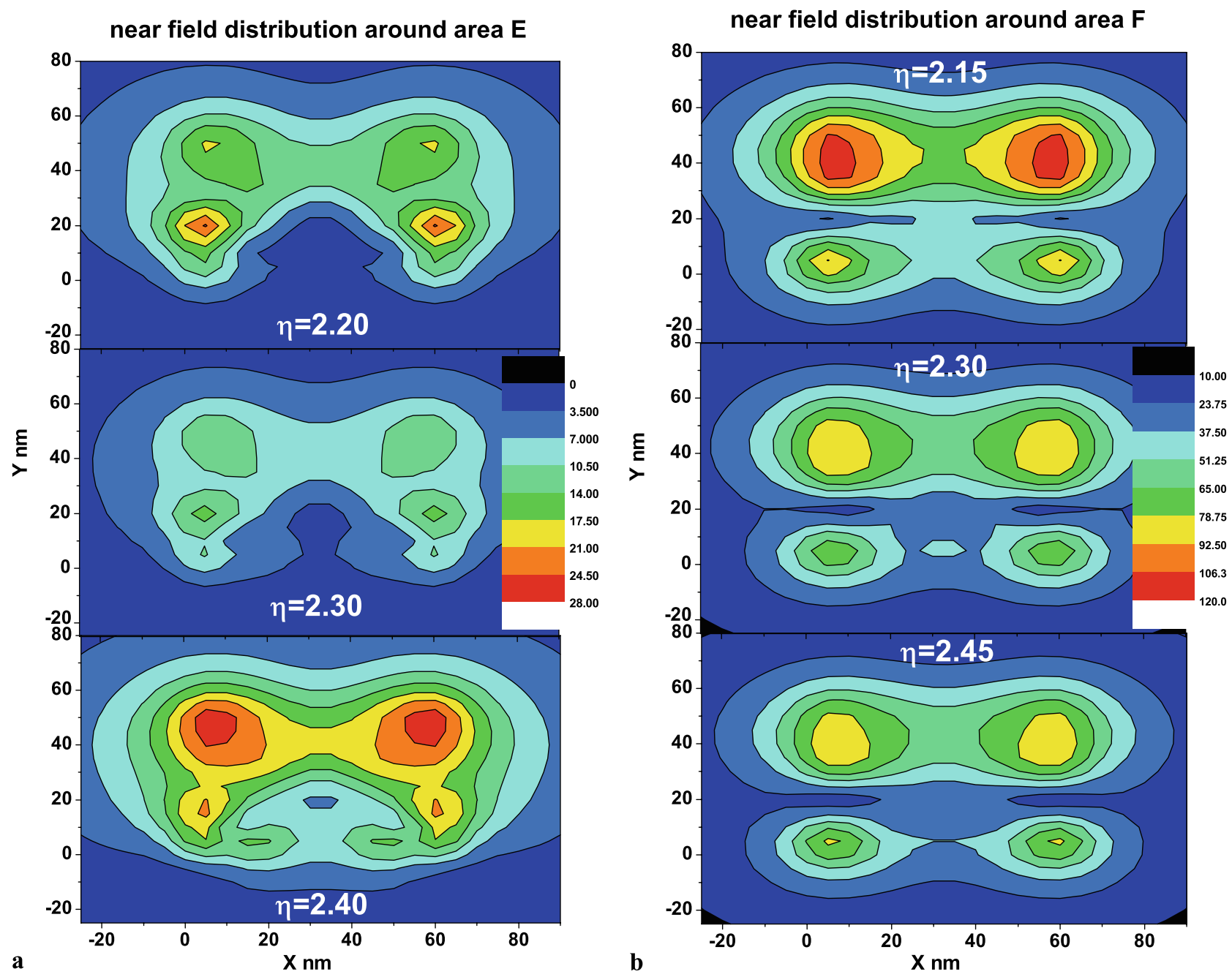

Fig. 5 Near field distributions above $10 \mathrm{~nm}$ of nanostrips around (a) area $E$ and (b) area $F$. Here the scale means the intensity of near field gathering at resonances caused by the same imaginary part $0.05 i$

we put all the resonance points with the large values of $C_{1}$ together, as well as the $C_{2}$ values corresponding to those $C_{1}$. Here we have drawn some lines to guide one's eyes. Another difference is that, except for the collective resonance region, in the other regions the $C_{2}$ values are also large. Different from the resonance flat region in the parallel case, at $\eta=2.3$, there is a concave $C_{1}$ curve. Though having the obvious difference from $\eta=2.15$, via $\eta=2.3$, to $\eta=2.45$, from the near field distributions around the concave area $F$ in Fig. 5(b), it seems that the fields are localized above the nanostrip 2. Almost the same explanation is found as in Fig. 5(a), i.e., the strongest field within the gap lead to the strong near field above the nanostrip 2 .

If the incident wavelength is fixed, theoretically, different geometrical nanoparticles will possess different resonant dielectric permittivities. In the spectral region, SPR interaction between different geometrical nanostructures with the same material has been studied [11]. Here, rather than consider two same nanostrips, we also explore the other geometrical sets, such as binary nanostructure including a nanostrip and a semi-nanoring. It is found that when $\eta<0$, i.e., one is a metal and another is a dielectric, the resonant behavior is almost the same as the above two nanostrips case. While, for $\eta>0$, instead of the previous one or two main resonances-dominated case, one strong resonance usually is destroyed into many weak ones, i.e., one branch is divided into many subbranches with small resonance capacity values. However, for symmetrical binary three nanostrips, resonance behavior is found to be similar to the two nanostrips case [21]. By carefully choosing the geometry and arrangement of each component, hybrid resonant structures can be used to design multi-resonance plasmonic structures.

If we consider real metals, the dielectric permittivity region should be mapped into the wavelength region. In the large wavelength region, a subwavelength structure will preserve the stable resonance spectra with respect to the di- 
Fig. 6 Absorption cross sections for $\mathrm{a} \mathrm{Au}-\mathrm{Ag}$ nanostrip system. (a) Parallel case and (b) perpendicular case. The same size and polarization parameters as before are used

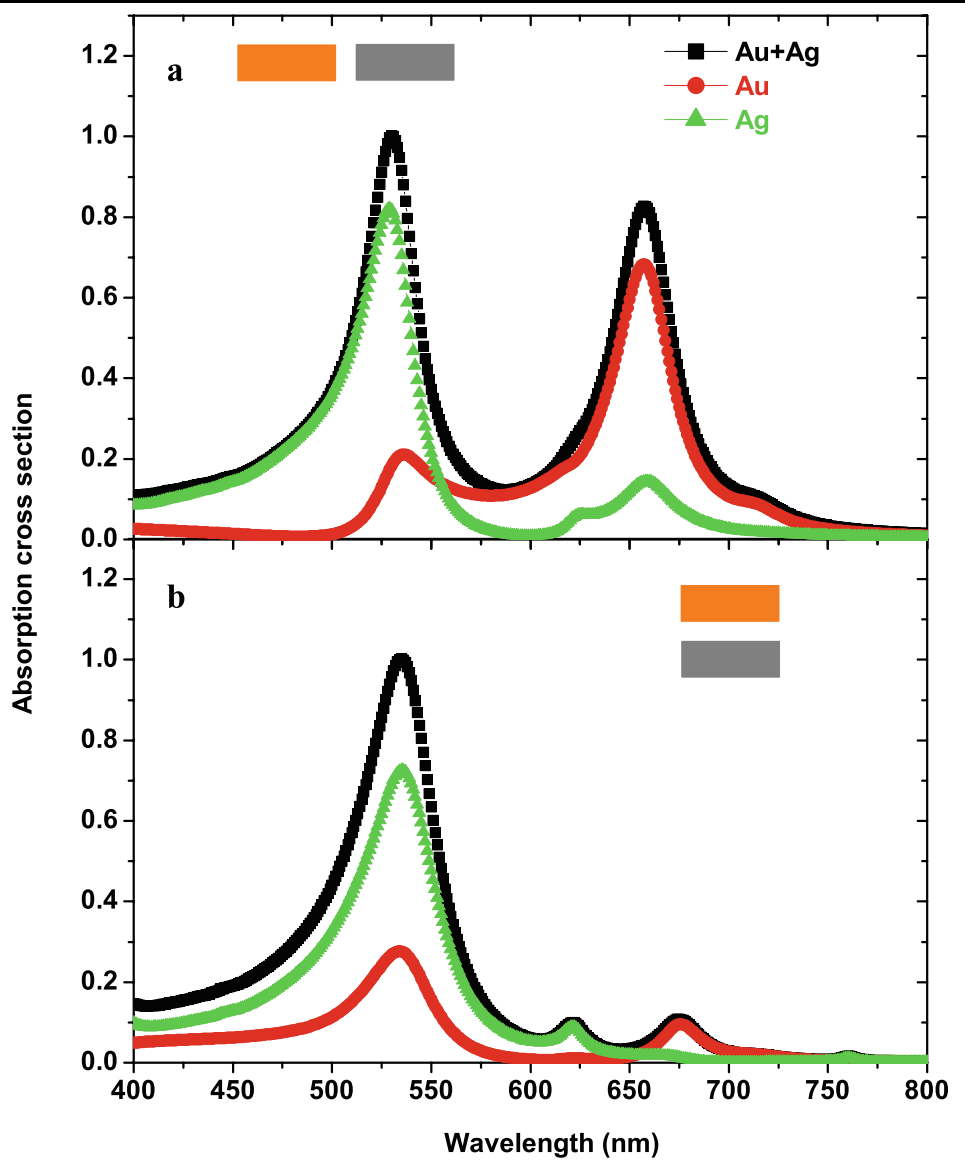

electric permittivity. For example, in [17], for the nanostrip $60 \times 20 \times 20 \mathrm{~nm}^{3}$, with varying the wavelength from $\lambda=500 \mathrm{~nm}$ to $\lambda=632.8 \mathrm{~nm}$, the dielectric constants of $\mathrm{Au}$ and $\mathrm{Ag}$ have large changes, such as $\epsilon_{\mathrm{Au}}(500)=-2.54$ and $\epsilon_{\mathrm{Au}}(632.8)=-11.78$. On the other hand, the resonance spectra with respect to the dielectric permittivity only have little modifications, namely, the resonance structure of surface plasmon is very stable with varying incident wavelength. Let us emphasize that, although in the previous discussion the wavelength is fixed at $\lambda=632.8 \mathrm{~nm}$ for illustration purpose, the corresponding resonance phenomena can be found to any part of the visible spectrum by carefully selecting the materials parameters [25].

Using the Green tensor technique [15], the absorption cross sections of $\mathrm{Au}-\mathrm{Ag}$ mixed nanostrip systems are calculated. There are two strong resonances at $\lambda=530 \mathrm{~nm}$ and $\lambda=660 \mathrm{~nm}$ for the parallel case and one strong resonance at $\lambda=535 \mathrm{~nm}$ for the perpendicular case, as shown in Fig. 6 . In the following we will see that these peaks are also well predicted by the Green matrix method. For the parallel case, at $\lambda=530 \mathrm{~nm}$, when $\eta=2.3$, the calculations indicate a strong resonance at $\epsilon_{\mathrm{Au}}=-4.78$ with $C_{\mathrm{Au}}=0.0102$ and $\epsilon_{\mathrm{Ag}}=-12.30$ with $C_{\mathrm{Ag}}=0.03818$. It means that the $\mathrm{Ag}$ nanostrip plays the main role in the absorption spectrum, in agreement with the result in Fig. 6(a), where the cross sec- tion of $\mathrm{Ag}$ is larger than the value of $\mathrm{Au}$. This agreement is also observed at $\lambda=660 \mathrm{~nm}$ in Fig. 6(a), where the results of the Green matrix method are $\epsilon_{\mathrm{Au}}=-14.47$ with $C_{\mathrm{Au}}=0.07168$ and $\epsilon_{\mathrm{Ag}}=-21.27$ with $C_{\mathrm{Ag}}=0.03394$, and at $\lambda=535 \mathrm{~nm}$ in Fig. 6(b), where $\epsilon_{\mathrm{Au}}=-5.31$ with $C_{\mathrm{Au}}=0.0739$ and $\epsilon_{\mathrm{Ag}}=-12.79$ with $C_{\mathrm{Ag}}=0.18766$. It is found that there are some differences between the predicted dielectric permittivities and the values of real metals. However, when we map these values into the wavelength region, there is only a $5 \sim 15 \mathrm{~nm}$ difference. The above results are also in line with the results of heterogeneous $\mathrm{Au}-\mathrm{Ag}$ dimers [27]. Hence we have verified the accuracy of our results using a completely different numerical approach.

Finally, we discuss the validity of numerical results without considering the loss of metal. It is noted that, for a fixed wavelength and a specific nanostructure, when we use GMM to predict those resonance dielectric permittivities $\epsilon=\epsilon^{\prime}+i \epsilon^{\prime \prime}$, the imaginary part $\epsilon^{\prime \prime}$ is not considered. Generally, when we consider surface plasmon resonances in optical wavelength, $\left|\epsilon^{\prime}\right| \gg\left|\epsilon^{\prime \prime}\right|$, which means that the imaginary part of the dielectric permittivity will not have a large influence in the resonance position. However, $\epsilon^{\prime \prime}$ determines the width of resonance and the intensity of the near field enhancement in some degree. In [17], by comparing GMM with Discrete dipole approximation (DDA) [26], 
a $20 \sim 50 \mathrm{~nm}$ difference in resonance was observed, indicated a good agreement between both methods. Recently, for the completely same nanostrips of $\mathrm{Au}$, results obtained from GMM without considering losses and DDA with considering losses indicate a $30 \mathrm{~nm}$ discrepancy in the resonance wavelength between $500 \mathrm{~nm}$ and $1000 \mathrm{~nm}$ [25]. We can therefore conclude that using GMM to predict the surface plasmon resonances is physically meaningful.

\section{Summary}

In this work, we have first extended the Green matrix method to two-component nanostructures. We have investigated the resonance combination of surface plasmons in two nanostrips. It is found that, when one nanostructure is metal and the other one is dielectric, the effect on the resonances is expressed as a very small shift. When both nanostructures are metal, the resonance properties are generally dominated by one metal or expressed as a collective resonance of both metals. We have also obtained the avoiding of crossing and mode transfer phenomena between resonance branches. The study of the resonance combination should provide the foundation for the design of hybrid plasmonic devices, such as plasmonic sensors and plasmonic waveguides.

Acknowledgements This work was supported by the National Natural Science Foundation of China under Grants Nos. 10874004, 10674009, 10821062 and National Key Basic Research Program No. 2007CB307001. The discussions with Prof. Xiaoyong Hu are appreciated.

\section{References}

1. A. Wokaun, J.P. Gordon, P.F. Liao, Phys. Rev. Lett. 48, 957 (1982)
2. S. Lali, S. Link, N.J. Halas, Nat. Photonics 1, 641 (2007), and references therein

3. P. Muhlschlegel, H.J. Eisler, O.J.F. Martin, B. Hecht, D.W. Pohl, Science 308, 1607 (2005)

4. J.R. Cole, N.J. Halas, Appl. Phys. Lett. 89, 153120 (2006)

5. A. Wokaun, J.G. Bergman, J.P. Heritage, A.M. Glass, P.F. Liao, D.H. Olson, Phys. Rev. B 24, 849 (1981)

6. E.M. Kim, S.S. Elovikov, T.V. Murzina, A.A. Nikulin, O.A. Aktsipetrov, M.A. Bader, G. Marowsky, Phys. Rev. Lett. 95, 227402 (2005)

7. M. Danckwerts, L. Novotny, Phys. Rev. Lett. 98, 026104 (2007)

8. Y. Lu, G.L. Liu, J. Kim, Y.X. Mejia, L.P. Lee, Nano Lett. 5, 119 (2005)

9. H. Tamaru, H. Kuwata, H.T. Miyazaki, K. Miyano, Appl. Phys. Lett. 80, 1826 (2002)

10. L. Gunnarsson, T. Rindzevicius, J. Prikulis, B. Kasemo, M. Kail, S.L. Zou, G.C. Schatz, J. Phys. Chem. B 109, 1079 (2005)

11. P.K. Jain, S. Eustis, M.A. El-Sayed, J. Phys. Chem. B 110, 18243 (2006)

12. B.M. Reinhard, M. Siu, H. Agarwal, A.P. Alivisatos, J. Liphardt, Nano Lett. 5, 2246 (2005)

13. P.K. Jain, W.Y. Huang, M.A. El-Sayed, Nano Lett. 7, 2080 (2007)

14. P.K. Jain, M.A. El-Sayed, Nano Lett. 7, 2854 (2007)

15. O.J.F. Martin, C. Girard, A. Dereux, Phys. Rev. Lett. 74, 526 (1995)

16. Y. Gu, K.W. Yu, H. Sun, Phys. Rev. B 59, 12847 (1999)

17. Y. Gu, L.L. Chen, H.X. Zhang, Q.H. Gong, EPL 83, 27004 (2008)

18. P.M. Morse, H. Feshbach, Methods of Theoretical Physics (McGraw-Hill, New York, 1953)

19. E.N. Economou, Green's Functions in Quantum Physics, 2nd edn. (Springer, Berlin, 1990)

20. Y. Gu, Q.H. Gong, Phys. Rev. B 67, 014209 (2003)

21. The further numerical calculations support our viewpoints, which will be published elsewhere

22. A.K. Sheridan, A.W. Clark, A. Glidle, J.M. Cooper, D.R.S. Cumming, Appl. Phys. Lett. 90, 143105 (2007)

23. A.W. Clark, A.K. Sheridan, A. Glidle, D.R.S. Cumming, J.M. Cooper, Appl. Phys. Lett. 91, 093109 (2007)

24. Y. Gu, Q.H. Gong, Phys. Rev. B 69, 035105 (2004)

25. J. Li, Y. Gu, F. Zhou, Z.Y. Li, Q.H. Gong, J. Mod. Opt. 56, 1396 (2009)

26. P.K. Jain, K.S. Lee, I.H. El-Sayed, M.A. El-Sayed, J. Phys. Chem. B 110, 7238 (2006)

27. G. Bachelier et al., Phys. Rev. Lett. 101, 197401 (2008) 